\title{
GEO-INFORMATICS IN AGRICULTURAL RESEARCH AND DEVELOPMENT
}

\author{
Uma Devi \\ Research Scholar, Andhra University, Visakhapatnam,A.P., India \\ umadadi1@gmail.com
}

Keywords: Geo-informatics, GIS, Remote sensing, Agriculture, Global Positioning Systems, Web Based Applications, Spatial Data, Databases

\begin{abstract}
A B S T RA C T
Geo-informatics is incorporated innovation for accumulation, change and age of data from coordinated spatial and non-spatial information bases. Remote detecting, Geographical Information Sciences (GIS), Global Positioning Systems (GPS), Relational Data Base Management Systems (RDBMS) are some of its critical fixings. It is an intense device for evaluation, observing, arranging and administration of agrarian innovative work. Administration of agrarian assets is a heap movement of protection practices and land/water assets went to expanding the sustenance generation. Significant increment in edit creation could be accomplished by bringing extra land under development, enhanced harvest administration innovation through utilization of high yielding, input responsive and push tolerant product assortments, enhanced nuisance control and in addition by expanding water system and manure inputs
\end{abstract}

Citation: Dadi Uma Devi (2018). Geo-Informatics In Agricultural Research And Development. International Journal of Advanced Multidisciplinary Scientific Research (IJAMSR ) ISSN:2581-4281 Vol 1, Issue4, June 2018, \#Art.215, pp48-58

\section{Introduction}

Geo-informatics is incorporated innovation for accumulation, change and age of data from coordinated spatial and non-spatial information bases. Remote detecting, Geographical Information Sciences (GIS), Global Positioning Systems (GPS), Relational Data Base Management Systems (RDBMS) are some of its critical fixings. It is an intense device for evaluation, observing, arranging and administration of agrarian innovative work. Administration of agrarian assets is a heap movement of protection practices and land/water assets went to expanding the sustenance generation. Significant increment in edit creation could be accomplished by bringing extra land under development, enhanced harvest administration innovation through utilization of high yielding, input responsive and push tolerant product assortments, enhanced nuisance control and in addition 


\section{International Journal of Advanced Multidisciplinary Scientific Research (IJAMSR) ISSN:2581-4281}

by expanding water system and manure inputs [4]. These data sources together with dependable data on I) existing area utilize and land under different harvests, ii) soil writes and degree of issue soils, iii) observing of surface water bodies (to decide water accessibility in water system frameworks) for ground water improvement and (iv) administration of common disasters [and so forth will empower detailing of suitable procedures to maintain the pace of rural advancement. This thus requires an all encompassing methodology, which must join here and now administration of horticultural assets at a small scale level with long haul worldwide viewpoints, keeping in perspective of financial and social condition of the general population. The part of room Geo-informatics in finding new assets for farming advancement for ideally dealing with the officially accessible assets, keeping in mind the end goal to augment horticulture creation is perceived worldwide and is observed to be exceptional potential.

Horticultural remote detecting including products and soils are very intricate. These complexities are because of the dynamic nature and intrinsic multifaceted nature of organic materials. So as to deal with these mind boggling issues, remote detecting innovation offers various favorable circumstances over conventional strategies for leading horticultural and other asset studies [3]. Favorable circumstances, incorporate, the potential for quickened overviews, ability to accomplish a brief view under moderately uniform lighting conditions, accessibility of multi-phantom information for giving extraordinary data, capacity of monotonous scope to delineate occasional and long haul changes and accessibility of symbolism with least twisting and so on. In this manner, it allows coordinate estimation of critical agro-physical parameters. Remote detecting of earth assets uses electromagnetic waves, which ranges from short wavelength ultraviolet through noticeable close infrared and warm infrared in the more drawn out wave length, dynamic radar and detached microwave frameworks. An incredible progression in utilization of PCs to this science is the improvement of capacity of putting away huge and changed data, running from authentic data and elevated photography to shuttle information, ground reference, and different types of subordinate information [5]. All these data is put away as profoundly valuable database/data framework. In this manner remotely detected information, and its inferred data have turned into a fundamental segment of farming administration framework in the nation. Utilizations of room, borne remote detecting information for expansive zone edit study was investigated in the USA under Corn Blight Watch Experiment (CBWE) in 1971 which was trailed by the vast number of trials/extensive scale remote detecting program. In a nation like India, with the immense geographic spread and extraordinary decent variety in its set up, the need to apply remote detecting innovation for national improvement was perceived amid mid 70's. The spearheading test was of coconut root- shrivel sickness utilizing shading infrared ethereal photography. Quantities of studies were led to methodological improvement here. Remote detecting exercises in India got an enormous lift with the dispatch of Indian Remote Sensing Satellite-1A (IRS 1A) in March 1988. India is moving quick being developed of new satellite frameworks and as of late propelled number of satellites devoted to the particular territory of utilizations, for example, OceanSat, CartoSat, ResourceSat and so on. Radar Imaging Satellite (RISAT), a microwave remote detecting mission with Synthetic Aperture Radar (SAR) working in C-band and having a $6 \times 2$ meter planar dynamic exhibit receiving 


\section{International Journal of Advanced Multidisciplinary Scientific Research (IJAMSR) ISSN:2581-4281}

wire in light of trans-beneficiary module engineering has been propelled as of late. Numerous innovative improvements, which happened in twentieth century added to the advancement of the idea of accuracy cultivating which incorporates GPS, GIS and high determination remote detecting satellite information. In following segments, commitments of this establishment are exhibited in this developing field.

Indian Agricultural Statistics Research Institute (IASRI) was built up on July 02, 1959 as an Institute of Agricultural Research Statistics. The order of the Institute is to embrace essential, connected and versatile research in Agricultural Statistics, to direct postgraduate and in-benefit instructional classes in Agricultural Statistics and Computer Applications, to give consultancy administrations, to go about as a store of data on Agricultural Statistics to examine. The foundation has been recognized as an Advanced Center of Excellence in instruction and preparing in Agricultural Statistics and Computer Applications. Aside from this organization likewise liaise with foundations of National Agricultural Research System (NARS), National Agricultural Statistical System (NASS), Department of Space (DOS) and so on., to aid the improvement and reinforcing of the nature of rural research and rural measurements through endeavor research and consultancy ventures. Organization is commending the Golden Jubilee year (July 03, 2008 July 02, 2009) of its establishment. This establishment likewise perceived capability of Geo-informatics innovations and started working toward an age of yield generation measurements since the mid nineties. Amid these years establishment has attempted a number of research ventures/contemplates and made huge commitments comprehensively in the regions of (I) Crop yield estimation (ii) Spatial stratification systems (iii) Small zone estimation, (iii) Spatial examining (iv) Spatial demonstrating (v) Classification methods (vi) Integrated overviews for sloping areas and (vii) Web GIS. Some of critical commitments of the establishment are quickly portrayed in following sub-areas

\section{Crop yield estimation}

Research on trim yield estimation has been taken up by the foundation since the start of the nineties. Goyal (1990) showed that remotely detected satellite ghastly information as vegetation records has been utilized to post-stratify the edited region into the territory of homogeneous harvest power and therefore enhanced estimators are proposed to evaluate the product yield utilizing remote detecting information alongside the ground specified yield estimation study information. The helpfulness of the proposed strategy has been exhibited by utilizing the Landsat (TM) satellite information and the product yield information from yield estimation study in light of harvest cutting trials from the Sultanpur region of Uttar Pradesh. Further, it has been watched that the Normalized Difference Vegetation Index (NDVI) when contrasted with Ratio Vegetation Index (RVI) can possibly segregate vegetation power and thus has the highest potential to be utilized as a part of harvest yield estimation reviews. An endeavor was additionally made to evaluate the impact of classification of units on the span of a post-strata and the post-stratified estimator of the product yield. The articulations for the predisposition and the fluctuation of the post-stratified estimator have been determined as far as the degree of misclassification. Since, otherworldly reflectance is an indication of coordinated impacts of all information sources like climate, soil and horticultural practices, in this manner it is normal that the phantom 


\section{International Journal of Advanced Multidisciplinary Scientific Research (IJAMSR) ISSN:2581-4281}

information can be utilized to advantage for trim yield anticipating. In this manner, endeavor was additionally made to think about the connection between wheat yield and the otherworldly parameters got through the handheld ghastly radiometers to investigate the handiness of unearthly information on trim yield determining.

Singh and Goyal (1993), Singh et al. (2000) and Singh and Goyal (2000) broadened the priority outcomes for estimation of wheat trim yield for locale Rohtak, Haryana is utilizing crop cutting examinations information for the year 1995-96 and satellite phantom information from the Indian Remote Sensing Satellite IRS-1B LISS II information for February 17, 1996. Post stratified estimator of harvest yield utilizing unearthly information as vegetation records NDVI and RVI for stratification have been acquired for the locale. The effectiveness of the post stratified estimator in light of NDVI and RVI contrasted with the typical estimator comes to 1.42 and 1.28 separately. This examination along these lines nearly affirms the discoveries of the prior investigation that the locale level estimator of harvest yield might be acquired by diminishing the quantity of product slicing trials to around 2/third without losing the accuracy accordingly bringing about extraordinary funds of cost. Further, two little region estimators of harvest yield, to be specific the immediate estimator and the engineered estimator have been created at level utilizing post stratification in view of NDVI. The standard mistake of both the immediate estimator and the engineered estimator at these levels is inside 5 for every penny and, not surprisingly the manufactured estimator is more productive when contrasted with the immediate estimator. Ibrahim (1992) examined the utility of the multi-date phantom information taken at chose transitional circumstances in the developing season, in a Markov anchor model to figure trim yield. A hand-held unearthly radiometer has been utilized for gathering the ghostly reactions from the test plots of wheat trim at fortnightly interims amid the development of the harvest. The phantom parameters saw at the distinctive development organizes and additionally the watched yield has been used to reproduce an unearthly populace alongside the relating yield in light of a stochastic model. Das (2004) proposed elective approach of product yield estimation utilizing multi determination satellite information. The endeavors were made to make utilization of satellite ghostly information and spatial inspecting method for edit real estate estimation, trim yield estimation and product yield anticipating, which includes the utilization of satellite information on coarse spatial determination, which is less expensive with bigger airborne scope. It was demonstrated that remotely detected satellite information can be utilized adequately as a territory outline for directing harvest yield estimation overviews. Fine determination information is expensive and airborne scope is less, though poor determination information has bigger ethereal scope with lesser cost. Since, ghostly reflectance is an indication of coordinated impacts of all data sources like climate, soil and agrarian practices, it is required to have a high connection with editing energy and henceforth the product yield. Since, estimation of yield utilizing diverse unearthly records has given distinctive assessments endeavors were made to join the estimators from various vegetation lists utilizing different casings inspecting estimation procedure. It has been discovered that significant pick up in proficiency is acquired in numerous edge inspecting gauges when contrasted with regular estimator utilizing single list. A typical approach in order of satellite information is 


\section{International Journal of Advanced Multidisciplinary Scientific Research (IJAMSR) ISSN:2581-4281}

directed most extreme probability grouping. In directed greatest probability grouping, every pixel is distributed with a specific subject contingent upon its advanced number. The nearness of the blended pixels is an irritation with performing characterization, in light of the fact that in the customary order methodology, a pixel is considered as a rudimentary unit for the examination. An elective technique for satellite information arrangement is proposed, which includes the utilization of otherworldly mark bend for preparing the satellite pictures to assess the fundamental class marks using fluffy grouping created by the pointer. The endeavor was likewise made to create determining model in view of phantom information and agree-meteorology

\section{Spatial sampling}

In agrarian studies regularly the parameter of intrigue is land in nature, i.e. the perceptions are needy through space, hence established insights can't be connected in that capacity. Reliance infers relationship and spatial reliance infer the nearness of spatial auto correlation. Since, in geological information 'contiguous units are frequently more indistinguishable than units that are far separated', it is alluring to misuse this data in the examining outlines. Along these line copy data halfway contained in regions as of now inspected can be kept away from. Another favorable position is that the testing expense can be streamlined without loosing the unwavering quality of the appraisals. An endeavor by Misra (2001) is being made to enhance the traditional review system for farming overviews with the assistance of spatial inspecting methodology. The capability of GIS to deal with different sorts of data through their geographic directions and Remote Sensing with its preference of wide zone scope, monotonous scope and succinct view have been misused for the investigation
[6]. An enhanced spatial examining procedure known as Contiguous Unit Based Spatial Sampling (CUBSS) Technique is proposed in this examination (Sahoo et al., 2006). The procedure consolidates estimate, measure alongside spatial contiguity of the units in the populace. The spatial relationship is assessed for assistant character which is utilized alongside the estimate, measure in appointing weights for the choice of the testing units. The likelihood of the determination of any unit is represented by these weights. The rule of test case is that the likelihood of choice of any unit increments as the separation of the units (region) officially chose increments. The example choice rule depends on the weights, representing spatial inconstancy and the size measure representing a real degree. Further, a reasonable fair estimator which considers the request of the draw is proposed for this circumstance. The examination is conveyed for customary grid, i.e. expecting the zone to comprise of customary units. Keeping in mind the end goal to handle the issue of inconsistency of the inspecting units, separate based neighbor is proposed. In view of these neighbors the changed equation for spatial connection is likewise recommended in this examination. For characterizing these neighbors, the idea of slacked variable and slacked arrangement is being utilized. A spatial inspecting method named as Distance Unit Based Spatial Sampling (DUBSS) is likewise proposed in this examination and its effectiveness is contrasted and the current ones and CUBBS strategy via completing an appropriate reproduction think about [7]. The proposed method performs significantly superior to the various systems.

Positioned Set Sampling (RSS) is found to give preferable outcomes over Simple Random Sampling (SRS) when positioning is simple and savvy, particularly 


\section{International Journal of Advanced Multidisciplinary Scientific Research (IJAMSR) ISSN:2581-4281}

in biology and rural overviews. After a basic audit of writing it has been discovered that there is a scarcely any procedure of RSS, which considers spatial reliance of perception produced by spatial factors. Kankure (2007) made an endeavor to create spatial positioned set examining system for the estimation of limited populace mean. Four inspecting outlines were proposed which considers the spatial connections of the areal testing units in the populace while choosing an example. s The proposed Spatial Ranked Set Sampling (SRSS) methods include the determination of extreme inspecting units in two phases. In the primary stage Random Spatial Clusters (RSC) of testing units in the populace are framed and in the second stage positioned set an example of indicated measure is being chosen. Test determination of the primary stage is finished by applying Dependent Areal-Unit Sequential Technique (DUST). This strategy depends on giving diverse probabilities of determination to the testing units such that closer units, or officially chose units in the example, get lesser likelihood of choice, while more remote units get higher likelihood of choice. It is one of the alluring attributes of an examining outline in spatially corresponded populace. The spatial part of the information is consolidated at this phase by partitioning the whole populace into Random Spatial Clusters (RSCs) by considering first stage units as the key units. The spatial groups are shaped based on the closest neighborhood approach as for haphazardly chose units. A specific unit will fall solely in a solitary spatial group, which has been framed by the key unit closest to it based on Cartesian separations figured utilizing scopes (La) and longitudes (Lo) of the areas. Having chosen the RSC, RSS was utilizing two methodologies i.e. (I) the whole positioned set example was chosen freely from each RSC and (ii) distinctive arrangements of the RSS are chosen autonomously from various RSC. Hence, in this examination some new and more effective testing procedures have been proposed which consider the spatial relationship show in the land units. The consequences of the investigation call attention to that, in spatial overviews, an impressive pick up in effectiveness of the estimators could be accomplished by utilizing separation based example choice techniques notwithstanding while applying these for complex testing plans, for example, RSS. The perplexing calculations associated with the determination system of separation based inspecting techniques could be tackled with the utilization of cutting edge processing and programming.

It is a verifiable truth that there is no target procedure for estimation of region under various yields in NorthEastern states because of normal issues existing in these districts. The north-eastern states, especially Meghalaya, principally comprises of sloping locale, with thick woodland cover. Other than this, the primary issue is its undulating geography and non-availability of immense region. Further, the relative rate territory under the harvests is less. For the most part terraced cultivating and Jhum development is rehearsed in these areas. In addition, these regions, especially Meghalaya, are secured by mists more often than not in a year. Along these lines it is hard to get cloud free pictures of these regions. Along these lines, utilization of remote detecting satellite information alone will most likely be unable to give solid data. Further, there are no cadastral maps and town limit maps existing for these districts. In spite of different states, in northeastern areas dependable data with respect to add up to a number of towns in each locale/square isn't accessible. Further, inside a towns add up to the number of agriculturists, number of fields 


\section{International Journal of Advanced Multidisciplinary Scientific Research (IJAMSR) ISSN:2581-4281}

claimed by every rancher, crops developed by the designers and so on are additionally not accessible in town records [8]. In this manner, the customary procedure of territory estimation isn't appropriate in these districts. Keeping this in see, an investigation (Sahoo et al., 2008) was taken up by Indian Agricultural Statistics Research Institute (IASRI), New Delhi in a joint effort with North-Eastern Space Applications Center (NESAC) Shillong and Space Applications Center (SAC) Ahmedabad in which philosophy was produced utilizing coordinated approach of remote detecting, GIS and ground review for estimation of the region under winter paddy trim in Meghalaya. The satellite information of IRS 1D, LISS III sensor has been utilized for this examination. Under this approach the territory under paddy has been gotten by normal arrangement strategy. There are two main considerations influencing the precision of the yield territory as got from the ordered satellite picture in the uneven locales: (I) Due to undulating geology of the district, misclassification and topographic geometry, there might be expansive contrasts of zone under harvest in the picture and real zone under product on the ground which may likewise bring about bigger degree of misclassification mistakes (ii) The region under paddy edit falling under slope shades or valleys may not be presented to the satellite sensor, as satellite sensors are sun-synchronous. Further, little paddy fields are not perceptible because of lower spatial determination of the LISS-III sensors. So as to redress the territory under paddy edit because of undulating geography and misclassification blunders, the connection between zone under paddy in the arranged picture and real zone under paddy trim on the ground has been built up. The region under paddy which has not been caught by the satellite sensor because of slope shades and constraints of spatial determination of the sensor has been amended by a reasonable example overview of the support made along the National Highway/State streets in GIS condition. Reasonable estimators were created to assess the region under paddy in this cradle zone. The vector layer of this cushion was overlaid on the satellite-ordered picture and the comparing region of the picture was extricated. Utilizing these appraisals, the zone under paddy in the whole locale was evaluated.

An examination was attempted in the Yamunanagar locale of Haryana State (Ahmad et al., 2003), to build up a GIS based system for recognizable proof of potential agro-ranger service zones. In this examination, vital elements in charge of development of agro-ranger service were recognized, appropriateness file utilizing Spatial-Analytic Hierarchy Process was built and was contrasted and the Composite advancement record. Another Objective Analytic Hierarchy Process (OAHP) technique was utilized to create appropriateness file for agro-ranger service of every town. This investigation endeavors to discover the potential agro-ranger service territories utilizing Geographic Information System (GIS) and proposed OAHP prompting Objective SpatialAHP to distinguish and rank regions that are reasonable for agro-ranger service, utilizing the measurable strategies engaged with the proposed OAHP and information contained in GIS maps. This examination is helpful for the agriculturists and in addition organizers. The investigation is useful for the social, financial and ecological improvement of the examination zone. The examination will likewise be useful in future research in the territory of agro-ranger service for different locales.

\section{Spatial modeling}




\section{International Journal of Advanced Multidisciplinary Scientific Research (IJAMSR) ISSN:2581-4281}

Rural fields are Spatial in nature. In the event that we consider profitability of the field as for certain yield gave different factors as consistent. It doesn't change unexpectedly starting with one field, then onto the next. The change is extremely steady and the neighboring fields have pretty much same structure. All in all product cutting analyses (CCE) are completed for yield estimation in choosing towns. It might be noticed that from trim cutting trial the estimation of creation at bring down the level like thesis, towns and so on is troublesome and inclined to huge mistakes because of little example measure. The use of spatial measurements in farming to enhance the forecast and estimation might be a helpful endeavor for little zone levels. Spatial qualities and CCE will help us in giving the better gauge and at bringing down level too. With the assistance of accessible writing in the field of spatial insights, it is conceivable to apply reasonable spatial models to foresee the creation surfaces, i.e. estimations of creation at each purpose of the guide, of the objective area. Field sizes of our nation are genuinely little, hence it is more suitable to use the phantom information as assistant data. An examination was attempted by Gupta (2002) to build up an incorporated approach for wheat edit yield estimation utilizing the student information on wheat trim yield from CCE alongside the satellite phantom information as vegetation lists i.e. Standardized Difference Vegetation record (NDVI). The utilization of remote detecting satellite information alongside the harvest yield information in view of CCE can extraordinarily enhance the productivity of harvest yield estimators at little region level.

Spatial measurements depends on the suspicions that close-by units are somehow related and tend to have comparable trait esteems. The traditional factual hypothesis depends on the supposition of autonomy of perceptions. Subsequently, traditional, measurable technique when connected to Geo-referenced information, neglect to catch the spatial reliance on the information. It is impossible to accept that the data is an area free while investigating the information of a character, which is land in nature, for example, efficiency of production, soil parameters and accessibility of ground water for the water system. Spatial information is information identified in the area of highlights alongside data identified by factors of intrigue. It is constantly alluring to use the information contained in the example to enhance the estimation of spatial measurements. Bayesian approach considers any earlier information of parameter/variable of intrigue.

Gupta (2007) influenced an endeavor to create spatial expectation to demonstrate under four unique circumstances i.e.

(i) using earlier data about parameters which is non-educational for known and obscure fluctuation

(ii) using earlier data about parameters which is educational for known and obscure fluctuation, (iii) utilizing earlier data about parameters as regular conjugate earlier for known and obscure change and (iv) utilizing fluffy approach for direct interim model for dubious characters under investigation. It was appearing in this examination through reenactment that Bayesian relapse investigation is constantly better than basic relapse investigation. This might be because of the way that the data contained in the example and additionally about the parameter of the model has been used in the estimation system. Further, there is a huge pick up in the accuracy if there should be an occurrence of geological factors when spatial impacts were considered in the 


\section{International Journal of Advanced Multidisciplinary Scientific Research (IJAMSR) ISSN:2581-4281}

estimation methodology under Bayesian system. It can be seen that variogram models assumes critical part in catching the spatial impact. Spatial Bayesian relapse demonstrates performs better when spatial impacts are fused through variogram models. The outcomes acquired through exponential and round variogram models were observed to urge when contrasted with different models.

Estimation Errors (ME) in informative factors of the traditional relapse show make the estimates of relapse coefficients one-sided and conflicting. In the event that, variable of intrigue is land in nature, relapse coefficients don't stay settled over space and common relapse investigation fails to assess the spatial area in its examination [3]. In this manner, another method called Geographically Weighted Regression (GWR) is utilized as a part of which evaluations of relapse coefficients depend on a neighborhood connection rather than worldwide relations among spatial factors of intrigue. Estimation of relapse coefficients when spatial illustrative factors with ME are settled or arbitrary in GWR show is relied upon to give productive gauges when contrasted with relates common relapse display. An endeavor has been made in this organization to propose a Functional Spatial Regression (FSR) show under ME and a Structural Spatial Regression (SSR) display under ME for estimation of relapse coefficients if there should be an occurrence of spatial factors. Illustrative factors under FSR show are thought to be settled while it is arbitrary in the event of SSR demonstrate. Altered evaluations of spatial relapse coefficients were proposed following Ordinary Least Squares (OLS), Generalized Least Squares (GLS), Maximum Likelihood Estimation (MLE) and Method of Moment Estimation (MME) approaches in both model structures. It has been appeared through spatial reenactment, that proposed estimators are fair-minded, reliable and nearly more productive than relating normal estimators. Four distinctive methodologies were taken after to fuse spatial impacts in the proposed model (Jha, 2009).

An investigation was attempted by Rai et al. (2004) in the region of Lalitpur in UP because of the way that this locale has been seen to have an extensive zone under a large portion of the land utilize arrangement classifications. It has been seen in this examination that nature of income records in the investigation zone, i.e. Lalitpur region is very dependable for a large portion of the typical nine overlays ordered land utilize classes [5]. The measurements of land utilize classes were limited to five more extensive classes, which can be distinguished by utilizing single time computerized information of Remote Sensing out of the over nine-overlap order can be effortlessly acquired utilizing RS. These measurements of land utilize classes got through RS could be utilized as assistant data in spatial/non-spatial models to get solid insights of various classes. The above models can be utilized to anticipate the measurements identified with these classes for nonstudied territory/towns of the regions. Henceforth, it is conceivable to create dependable land utilize insights at any littler level, i.e. Panchayat/square/thesis utilizing above models. Keeping in mind the end goal to assess spatial reliance of the neighboring units, the traditional examining procedure approach is being altered with the end goal that the probabilities of choosing neighboring units, once a specific unit are chosen in the example, turns out to be less when contrasted with far off areal units. The best fitted spatial model for each class of land utilize was observed to appear as something else, contingent upon the spatial appropriation of the land 
utilize class patches of land in the region. The forecast of territory under various land utilize classifications secured under nine overlay arrangement in light of satellite information utilizing spatial model is by all accounts very attractive.

Aside from these investigations quantities of research papers were distributed in national and global diaries by the scientists of the organization. A portion of the essential commitments in such manner are Singh et al. (1992), Singh and Ibrahim (1996), Singh et al. (2002), Sahoo et al. (2005), Sahoo et al. (2006), Rai et al. (2007), Ahmad et al. (2007), and so forth

\section{References}

1. Ahmad, T., Singh R., Rai, A. and Kant, A. (2007). Model for prediction of area under Yamunanager district of Haryana. Indian. J. Ag. Sci., 77(1), 43-45.

2. Ahmad, T., Singh, R.and Rai, A. (2003). Development of GIS based technique for Identification of Potential Agro-forestry area. Project Report, IASRI, New Delhi.

3. Dadi, Sanyasinaidu. "USE OF GIS IN HYDROLOGICAL INVESTIGATIONS." INTERNATIONAL JOURNAL OF MULTIDISCIPLINARY ADVANCED RESEARCH TRENDS.(2015):

Web. <https://www.researchgate.net/publication/321 069487_USE_OF_GIS_IN_HYDROLOGICAL_INV ESTIGATIONS>.

4. Dadi, Sanyasinaidu. "An Importunate Role of GIS in Indian Retail Industry." MAT Journals-Journal of Remote Sensing GIS \& Technology. (2017): Web.<http://www.matjournals.in/index.php/JORSGT /article/view/2110>.

5. Dadi, Sanyasinaidu. "Remote Sensing and Geographic Information System for Jungle Administration." MAT -matjournals-JOURNAL OF REMOTE SENSING GIS \& TECHNOLOGY (2017):
Web.<http://matjournals.in/index.php/JoADC/article /view/2128>.

6. Dadi, Sanyasinaidu. "GIS and Remote Sensing as Tool to Develop Applications for Natural Resource Management." MAT -matjournals-JOURNAL OF REMOTE SENSING GIS \& TECHNOLOGY. (2017). Web. <http://www.matjournals.in/index.php/JORSGT larticle/view/2101>.

7. Dadi, Sanyasinaidu. "GIS and Remote Sensing For Site Specific Farming Area Mapping." MATmatjournals-Journal of Analog and Digital Communications. (2017):

Web. <http://matjournals.in/index.php/JoADC/article /view/2126>.

8. Dadi, Sanyasinaidu. "UNDERSTANDING THE CONCEPT OF VIRTUAL GLOBE FOR A GIS PERSONNEL." INTERNATIONAL JOURNAL OF MULTIDISCIPLINARY ADVANCED RESEARCH TRENDS. (2015):

Web. $<$ http://ijmart.in/PreviousIssues/Sep2015/4.pdf>.

9. Dadi, Sanyasinaidu. "GIS Applications to Smart Cities." International Journal of Advanced Multidisciplinary Scientific Research(IJAMSR) Volume 1,Issue 1, February (2018).

10. Dadi SanyasiNaidu, and Peddada Jagadeeswara Rao. "Study on Sustainable Management of Groundwater Resources in Greater Visakhapatnam Municipal Corporation, Visakhapatnam District, India-A Hydro Informatics Approach.” Springer Series in Geomechanics and Geoengineering Proceedings of International Conference on Remote Sensing for Disaster Management, 2018, pp. 719 728., doi:10.1007/978-3-319-77276-9_64.

11. Das, S.K. (2004). Application of multiple frame sampling technique for crop surveys using remote sensing satellite data. A Ph.D. thesis submitted to P.G. School, IARI, New Delhi.

12. Goyal, R.C. (1990). Use of Remote Sensing Planning of Agricultural Surveys. Project Report, IASRI, New Delhi. 
13. Gupta, N.K. (2002). Applications of spatial models in estimation of wheat production in rohtak distrct of haryana. Unpublished M.Sc. thesis submitted to P.G. School IARI, New Delhi.

14. Gupta, N.K. (2007). On spatial prediction modeling. Unpublished Ph.D. thesis submitted to P.G.School IARI, New Delhi.s

15. Ibrahim, A.E.I. (1992.). Use of remote sensing data in a markov chain model for crop yield forecasting. Project report IASRI, New Delhi.

16. Jha, D.N. (2009). A study an spatial regression model under measurement area framework. Unpublished Ph.D thesis submitted to PG. School. IARI, New Delhi.

17. Kankure, A.K. (2007). Small area estimation of crop yield at block level using satellite data. Unpublished Ph.D. thesis submitted to P.G. School, IARI, New Delhi.

18. Misra, P. (2001). Applications of spatial statistics in agricultural surveys. Unpublished Ph.D. thesis, P.G.School, IARI, New Delhi.

19. Rai, A., Gupta, N.K. and Singh, R. (2007). Small area estimation of crop production using spatial models. Mod. Asst. Stat. Apl., 2(2), 89-98.

20. Rai, A., Srivastava, A.K., Singh, R., and Jain, V.K. (2004). A study of land use statistics through integrated modelling using geographic information system. IASRI, New Delhi Publication.

21. Sahoo, P.M., Rai, A., Singh, R., Handique, B.K. and Rao, C. S. (2008). Development of Remote Sensing based Methodology for collection of Agricultural Statistics in Megahalaya. Project Report IASRI, New Delhi.

22. Sahoo, P.M., Rai, A., Singh, R., Handique, B.K. and Rao, C.S. (2005). Integrated approach based on remote sensing and GIS for estimation of area under paddy crop in north-eastern hilly region' J. Ind. Soc. Agric. Stat., 59(2), 151-160.

23. Sahoo, P.M., Singh, R. and Rai, A. (2006) Spatial sampling proceduse for agricultural surveys using geogeaphical infoemation system. J. Ind. soc. Agril. Stat. 60 (2) 134-143.

24. Singh R., Goyal R.C., Pandey L.M. and Shah S.K. (2000) Use of remote sensing technology in crop yield estimation survey-ii. Project report IASRI, New Delhi.

25. Singh, R. and Goyal, R.C. (1993). Use of remote sensing technology in crop yield estimation surveys. Project Report, IASRI, New Delhi.

26. Singh, R. and Ibrahim, A.E.I. (1996). Use of spectral data in markov chain model for crop yield forecasting.

27. Singh, R. and Goyal, R.C. (2000) Use of remote sensing technology in crop yield estimation surveys. Project Report, IASRI, New Delhi.

28. Singh, R. Semwal, D.P., Rai, A. and Chhikara, R.S. (2002). Small area estimation of crop yield using remote sensing satellite data. Int. J. Rem. Sens., 23(1), 49-56.

29. Singh, R., Goyal, R.C., Saha, S.K. and Chhikara, R.S. (1992). Use of satellite spectral data in crop yield estimation surveys. Int. J. Rem. Sens., 13(14), 2583-2592. 